\title{
Confidence Appraisals Protect against Anxiety in Response to a Transient Stressor
}

\author{
Alyson K. Zalta, Dianne L. Chambless \\ Department of Psychology, University of Pennsylvania, Philadelphia, USA \\ Email: zalta@sas.upenn.edu
}

Received March 16 ${ }^{\text {th }}$, 2012; revised April 10 ${ }^{\text {th }}$, 2012; accepted May $12^{\text {th }}, 2012$

\begin{abstract}
Research suggests that cognitive appraisals regarding personal control and existential beliefs are important factors in the development of anxiety. These include mastery, generalized self-efficacy, locus of control, self-esteem, attributional style, and optimism. It remains unclear, however, whether these appraisals add to the prediction of anxiety beyond what can be determined by individuals' generalized tendency to respond with anxiety in stressful situations. Moreover, researchers have begun to question whether measures of these appraisals represent truly distinct constructs or whether they are merely redundant aspects of a broad higher order construct. We sought to determine the empirical overlap of these six cognitive appraisals known to correlate with anxiety and whether identified factor(s) prospectively predicted anxiety in response to an ecologically valid stressor. The appraisals loaded onto one factor reflecting confidence in one's coping ability. This confidence factor significantly predicted future state anxiety after controlling for baseline levels of trait anxiety and other possible confounds. Results suggest that these cognitive appraisals may be causally linked to anxiety and used to identify those at risk.
\end{abstract}

Keywords: Anxiety; Prospective; Longitudinal; Risk Factor; Cognitive Appraisal

\section{Introduction}

Anxiety disorders pose a substantial burden to both individuals and society, affecting approximately $29 \%$ of individuals in their lifetime (Kessler et al., 2005) and costing an estimated $\$ 42.3$ billion annually in 1990 (Greenberg et al., 1999). Considering the significant public health and economic burden posed by anxiety disorders, researchers have called for greater research efforts to elucidate etiological risk processes that can inform intervention strategies (Zvolensky, Schmidt, Bernstein, \& Keough, 2006). Historically, behavioral learning approaches served as the predominant etiological model for anxiety disorders; however, these theories suffered criticism as it became clear that this model could not account for the large degree of individual variation in anxiety after fearful learning experiences (Mineka, 1985). To resolve these concerns, contemporary learning approaches incorporate the concept of diatheses that make individuals more or less susceptible to stressful experiences (Mineka \& Zinbarg, 2006). These diatheses could represent a wide range of risk factors from genetic vulnerability to experiential learning history.

Cognitive appraisals ${ }^{1}$ have been identified as a potentially important factor in determining how individuals respond to stress and the subsequent development of anxiety. According to the stress-appraisal-coping framework proposed by Lazarus and Folkman (1984), when an individual is faced with a stressor, they make primary appraisals as to whether the stressor is a negative threat or a positive challenge and secondary appraisals regarding their ability to cope with the stressor. Anxiety is thought to occur when primary appraisals of the significance of

\footnotetext{
${ }^{1}$ We use the term cognitive appraisals to refer to constructs that represent stable ways of thinking about oneself, the world, and the future that affect anxious responding (Clark \& Beck, 2010).
}

a stressful event exceed secondary appraisals of one's coping ability. According to this theory, both person factors and situation factors influence individuals' appraisals. Person factors include individual's beliefs about their personal control (i.e., the extent to which people are confident that they can control the environment and important outcomes in their lives) and existential beliefs (i.e., beliefs that enable people to create meaning out of life and maintain hope). Individuals who perceive themselves as having control over their outcomes and who generally believe that their outcomes will be positive are less likely to experience anxiety because they are more likely to be confident in their coping ability and are less likely to view a stressor as threatening.

Studies examining various populations have shown that individuals with a sense of control and optimism are less likely to experience anxiety (e.g., Archer, 1979; Scheier, Carver, \& Bridges, 2001). Moreover, evidence suggests that measures of perceived control and optimism prospectively predict psychological distress following major life stressors (Bárez, Blasco, Fernández-Castro, \& Viladrich, 2009; Ben-Zur \& Zeidner, 1988; Dew et al., 1997; Dew et al., 2004; Dew et al., 1994; Gurung, Dunkel-Schetter, Collins, Rini, \& Hobel, 2005; Scheier, Carver, \& Bridges, 2001; van Jaarsveld, Ranchor, Sanderman, Ormel, \& Kempen, 2005; Windsor, Anstey, Butterworth, Luszcz, \& Andrews, 2007). These studies indicate that cognitive appraisals assessing one's personal sense of control and hope for the future may be important causal diatheses for anxiety. However, the majority of longitudinal studies have examined these appraisals as predictors of anxiety without controlling for preexisting levels of trait anxiety. It is possible that these appraisals do not add any predictive value beyond what is accounted for by individuals' general tendency to respond with anxiety in stressful situations. If this is the case, then the as- 
sessment of cognitive appraisals would not provide incremental information as to who is likely to develop anxiety, even though they might still contribute to the development of the chronic tendency to be anxious.

Researchers have also begun to question whether different cognitive appraisal measures that assess personal control and optimism are truly distinct or whether they are redundant aspects of a broad higher order construct (cf. Judge, Locke, \& Durham, 1997). Although it appears at face value that measures assessing a sense of control (e.g., mastery, self-efficacy), self-worth (e.g., self-esteem), and positive hope for the future (e.g., optimism) are separate constructs, it is possible that these traits may be empirically indistinguishable. For example, Judge and colleages (2002) showed that self-esteem, neuroticism, locus of control, and generalized self-efficacy form a single latent factor and reveal poor discriminant validity. By contrast, Scheier and colleagues (1994) showed that optimism continues to predict depression after controlling for the effects of selfesteem and self-mastery. Using different terminology to identify the same construct within various domains can be detrimental because researchers fail to consider these alternate literatures to inform their research and may engage in redundant efforts. However, it is equally problematic to combine seemingly similar traits that are in fact distinct constructs because this adds error to data analysis and obscures accurate interpretation. For example, investigators may conclude that findings in the field are inconsistent when in fact the target variable reflects multiple unique concepts.

To address these concerns, we sought examine the latent structure underlying six cognitive appraisals known to predict anxiety including measures that assess personal control and positive expectancies: mastery, generalized self-efficacy, locus of control, attributional style, self-esteem and optimism. Although it is true that other appraisals may fit into the stressappraisal-coping framework, these six constructs were selected for investigation because they all have well-validated measures that have been widely used in research on anxiety. We then sought to examine whether any identified factor(s) predicted future anxiety after controlling for individuals' general tendency to respond to stressors with anxiety (i.e., trait anxiety).

According to Lazarus and Folkman's theory (1984), cognitive appraisals not only impact individuals who experience a major life stressor, but also influence how people respond to the hassles that they experience in their daily life. Moreover, research suggests that measures of hassles, or more minor daily stressors, are better predictors of psychological symptoms than measures of life events (Monroe, 1983). Thus, we sought to examine whether cognitive appraisals play an important role in determining how individuals respond to the common stressors they are likely to face. Evidence suggests that for college students, testing situations are an important source of stress and anxiety (Souza, Samaraweera, \& Frye, 2009). We measured levels of state anxiety among college undergraduates in the 24 hours prior to a psychology exam to determine the role of cognitive appraisals in predicting anxiety in this salient and ecologically valid context. The longitudinal design of this study enabled us to control for preexisting levels of trait anxiety to establish whether cognitive appraisals create vulnerability for future anxiety independent of the general tendency to be anxious. Because it is plausible that actual (as opposed to perceived) lack of ability contributes to students' concerns about their performance and thereby their anxiety, we also examined the contribution of Scholastic Aptitude Test (SAT) scores as a potentially confounding variable.

\section{Method}

\section{Participants and Procedure}

Participants were undergraduates enrolled in Introduction to Psychology, Abnormal Psychology, or Social Psychology at the University of Pennsylvania during the Spring and Fall semesters of 2008. The overall sample $(N=337)$ included 132 men and 205 women ranging in age from 18 to 28 years old $(M=$ 19.3 , SD $=1.4)$. The racial composition of the sample was 58.5\% White, 25.2\% Asian, 5.9\% Latino, 5.3\% African American, $0.3 \%$ American Indian or Alaskan Native, 0.3\% Native Hawaiian or Pacific Islander, and 4.5\% Other or unknown.

After providing informed consent, participants completed two sets of online computer questionnaires. Those who completed both surveys received research credit to meet requirements for their undergraduate psychology course. At Time 1, students completed the six cognitive measures as well as a measure of trait anxiety and provided their SAT scores. This initial survey was completed at least two weeks prior to the students' scheduled psychology exam for the class in which they were enrolled. Students were re-contacted 24 hours prior to their scheduled psychology exam to complete the Time 2 survey, which included a measure of state anxiety. Only students who completed the Time 2 survey before their scheduled exam time were included in the final sample so as to capture their anxiety about a future event. Of the 337 students included in the baseline sample, 38 were lost to follow-up. This study was approved by the Human Subjects Committee of the University of Pennsylvania's Institutional Review Board.

\section{Measures}

Demographic and Course Information. At baseline, participants were asked to report their year in school, undergraduate major, SAT scores, parents' educational level, and how motivated they were to do well in their psychology course on a 7-point Likert-type scale (from not at all motivated to extremely motivated). At follow-up, students were asked to use the same scale to indicate how motivated they were to do well on the exam and to report how many other exams they had that week. On average, students reported being highly motivated to do well in their psychology course $(M=5.91, S D=1.12)$ and highly motivated to do well on their psychology exam $(M=$ $5.86, S D=1.21$ ); on both motivation questions, over two-thirds of students reported either a 6 or 7 .

Attributional Style Questionnaire (ASQ; Peterson et al., 1982). The ASQ is composed of 12 different hypothetical situations, consisting of 6 good events and 6 bad events. Individuals are asked to rate whether they see each event as caused by internal versus external, stable versus unstable, and global versus specific causes. Composites of internal, stable, and global attributions are created separately for positive and negative events, and the final summary score represents the difference between the composite scores for positive and negative events. High scores represent more beneficial attributional styles. Internal, stable, and global attributions for negative events are associated with anxiety (e.g., Ahrens \& Haaga, 1993). Cronbach's alpha in the current sample was .80 for positive events and .75 for negative events. 
Generalized Self Efficacy Scale (GSE; Schwarzer \& Jerusalem, 1995). The GSE is a 10-item scale designed to assess "the belief in one's competence to cope with a broad range of stressful or challenging demands" (Luszczynska, Scholz, \& Schwarzer, 2005: p. 439). Studies have consistently demonstrated a relationship between higher levels of generalized selfefficacy and better physical and mental health (Luszczynska et al., 2005). Cronbach's alpha was .87.

Life Orientation Test-Revised (LOT-R; Scheier et al., 1994). The Life Orientation Test was developed to assess individual differences in dispositional optimism or the generalized expectancies of good versus bad outcomes. The revised version (LOT-R) includes 10 items with 6 scored questions and 4 filler items. This measure is consistently correlated with measures of physical and psychological well-being (Scheier \& Carver, 1992). Cronbach's alpha in the current sample was .80.

Rosenberg Self-Esteem Scale (RSE; Rosenberg, 1965). This scale is the most commonly used measure of self-esteem, designed to assess an individual's global positive or negative attitude towards the self. Respondents are asked to rate themselves on 10 items, such as "I feel that I have a number of good qualities" on a scale from 1 (strongly disagree) to 5 (strongly agree). There are considerable data to support the validity of this scale (e.g., GrayLittle, Williams, \& Hancock, 1997). Research indicates that low self-esteem prospectively predicts anxiety (Lee \& Hankin, 2009). Cronbach's alpha in the current sample was .90.

Rotter's Locus of Control Scale (LOC; Rotter, 1966). The LOC is a 29-item forced-choice scale in which individuals are asked to select from two options reflecting internal or external locus of control. Individuals with a high internal locus of control believe that events result primarily from their own behavior and actions. Those with a high external locus of control believe that powerful others, fate, or chance primarily determine events. High scores represent high levels of externality. External control scores have been found to correlate with higher levels of anxiety in a variety of sample populations (Archer, 1979). Kuder-Richardson 20 coefficient in this sample was .73.

Self-Mastery Scale (SMS; Pearlin \& Schooler, 1978). The SMS is a widely used measure that assesses "the extent to which people see themselves as being in control of the forces that importantly affect their lives” (Pearlin, Menaghan, Lieberman, \& Mullen, 1981: p. 340). The scale contains 7 items rated on a 4-point scale from 1 (strongly disagree) to 4 (strongly agree). The measure has demonstrated the ability to longitudinally predict treatment outcome for patients with panic disorder and agoraphobia (Bowen, South, Fischer, \& Looman, 1994). Cronbach's alpha in the current sample was .80.

State-Trait Anxiety Inventory (Spielberger, Gorsuch, Lushene, Vagg, \& Jacobs, 1983). Trait anxiety refers to a stable tendency to experience anxiety in anticipation of seemingly threatening situations. State anxiety refers to transient emotional arousal and apprehension. These constructs are measured separately with 20 statements each. The trait anxiety scale (STAI-T), measured at baseline, asks participants to report how they generally feel. The state anxiety scale (STAI-S), measured at follow-up, asks respondents how they feel "right now, at this moment." Research has demonstrated that the STAI-T is useful for identifying individuals with clinical levels of anxiety and the STAI-S is a sensitive indicator of changes in transitory anxiety (Spielberger et al., 1983). The STAI-T is also a strong predictor of state levels of anxiety (e.g., $r=.46$ in the present sample). In the current sample, Cronbach's alpha was .92 for the STAI-T and .94 for the STAI-S.

\section{Results}

\section{Preliminary Analyses}

Before conducting the primary analyses, we first examined whether demographic variables and potential confounds were associated with state anxiety at Time 2 . Correlations were calculated for continuous variables, independent samples $t$-tests were calculated for binary variables, and regressions using dummy variables were calculated for nominal variables with more than two categories. The following variables were unrelated to state anxiety: age, year in school, semester the data were collected, which psychology course students were enrolled in, whether they were a psychology major, father's educational status, mother's educational status, motivation to do well in the course, motivation to do well on the test, and the number of days between baseline and follow-up assessments. All effects were small. Notably, total SAT scores were unrelated to state anxiety in the 24 hours prior to a psychology exam $(r=.05, p=.50)$.

Several other variables demonstrated significant relationships with state anxiety at follow-up. Women reported higher state anxiety $(t(293)=-2.66, p=.008, d=-.31)$ compared to men, and Asians reported higher state anxiety $(t(291)=-2.96, p$ $=.003, d=-.39$ ) compared to Whites. The number of other tests that participants had in the same week was also associated with state anxiety $(r=.12, p=.04)$. These three variables were controlled in prediction analyses.

Additional tests demonstrated that those who were lost to follow-up did not differ from those who participated in the follow-up assessment on any demographic variables.

\section{Factor Analysis}

The six measures were assessed using Bartlett's chi-square criteria (Geweke \& Singleton, 1980); this test was significant ( $p$ $<.001$ ), rejecting the likelihood that the variables are uncorrelated and indicating that factor analysis is appropriate with this dataset. A principal factor analysis was then conducted to determine the latent structure of these cognitive appraisal variables. This analysis resulted in a single factor (eigenvalue = 2.56) with all scales loading onto the first factor (all factor loadings $\geq .40$ ). No scales loaded on any additional factors (all factor loadings $<.20$ ) and the eigenvalue for the second factor was 0.06 . Thus a single common factor was determined to be the best solution. This factor appears to represent general confidence in oneself and the future, that one is worthy and capable of achieving success and that good things will come from life. To capture this factor in a single measurement, a factor score was calculated using a regression scoring method with a single factor solution. The means, standard deviations, correlation matrix, and factor structure are presented in Table $\mathbf{1}$.

\section{Longitudinal Prediction of State Anxiety}

We then sought to determine whether this confidence construct is an important prospective predictor of anxiety. The correlation between the factor score and Time 2 state anxiety revealed a medium to large effect $(r=-.43, p<.0001)$. A multiple regression was then conducted predicting Time 2 state anxiety from the following predictors: Time 1 trait anxiety, sex, 
Table 1.

Descriptive statistics, correlations, and factor structure of six cognitive appraisal measures.

\begin{tabular}{lcccccccccc}
\hline & Mean & SD & SMS & GSE & LOC & ASQ & RSE & LOT-R & Factor loading & Communality \\
\hline SMS & 21.97 & 3.11 & & .67 & -.48 & .38 & .65 & .68 & .72 & .54 \\
GSE & 31.48 & 4.27 & .56 & & -.43 & .49 & .57 & .55 & .69 & .48 \\
LOC & 12.05 & 4.07 & -.37 & -.34 & & -.41 & -.33 & -.44 & -.47 & .25 \\
ASQ & 3.04 & 2.87 & .30 & .40 & -.31 & & .49 & .52 & .54 & .32 \\
RSE & 21.17 & 5.11 & .55 & .50 & -.27 & .41 & & .71 & .73 & .55 \\
LOT-R & 14.62 & 4.05 & .54 & .46 & -.34 & .41 & .60 & & .73 & .54 \\
\hline
\end{tabular}

Notes: SMS = Self-Mastery Scale; GSE = Generalized Self-Efficacy Scale; LOC = Locus of Control; ASQ = Attributional Style Questionnaire; RSE $=$ Rosenberg Self-Esteem Scale; LOT-R = Life Orientation Test-Revised. Zero-order correlations are reported below the diagonal. Disattenuated correlations are reported above the diagonal.

Asian ethnicity, the number of other tests participants had in the same week at Time 2, and the factor score. Trait anxiety and other covariates were first entered in the model, the factor score was then entered in the model in a second block to determine the incremental predictive validity of this factor. The results of these analyses are presented in Table 2 . The overall regression model was significant $(p<.0001$ ), explaining $26.7 \%$ of the variance in Time 2 state anxiety. The confidence factor score ${ }^{2}$ significantly added to the prediction of state anxiety after controlling for Time 1 trait anxiety and other possible confounds.

\section{Discussion}

Results indicated that the six cognitive appraisals assessed in this study were all indicators of a single higher order factor which we describe as confidence in one's coping ability and the future based on the framework proposed by Lazarus and Folkman (1984). The latent association among these measures suggests that researchers should consider these alternate research literatures to aid in the accumulation of findings and avoid redundant research efforts. Investigators should especially look to these other literatures when a paucity of studies exists using a given construct. Although investigators may be wary of integrating studies that utilize different terms, our data suggest that empirically, this may be no different from integrating studies that use different measures of the same construct. Judge and colleagues (2002) reported that meta-analytic correlations between self-esteem and generalized self-efficacy were similar to or even exceeded typical correlations between different measures of the same construct. Thus, investigators should consider building off of rather than replicating research conducted with one of these cognitive appraisals.

Having established this latent factor, we then sought to determine whether these variables prospectively predicted state anxiety in the 24 hours prior to an undergraduate psychology exam, a salient and common stressor for college students. Results showed that the factor score had a moderate to large association with later state anxiety and prospectively predicted state anxiety above and beyond the contribution of prior levels of trait anxiety and potential confounds. Examination of the standardized betas indicates that the confidence factor was the second largest predictor of state anxiety after trait anxiety and provided a greater contribution to the prediction of state anxiety than biological sex, a well known risk factor for anxiety. By contrast, SAT scores were unassociated with anxiety, indicating

\footnotetext{
${ }^{2}$ The factor score was also a significant predictor of anxiety in the regression
} model when calculated with unit weighting of the six measures.
Table 2.

Hierarchical multiple regression of Time 1 variables predicting state anxiety at Time 2 .

\begin{tabular}{cccc}
\hline & & $\beta$ & $\Delta R^{2}$ \\
\hline Block 1 & Sex & $.135^{* *}$ & $.257^{* * *}$ \\
& Asian & $.087^{\dagger}$ & \\
& Other tests & $.139^{* * *}$ & \\
& Trait anxiety & $.318^{* * *}$ & \\
Block 2 & Factor score & $-.171^{*}$ & $.011^{*}$ \\
\hline
\end{tabular}

Notes: $N=293$. For sex, male $=0$, female $=1$. $\beta$ represents the standardized betas with all variables entered into the model. $\Delta R^{2}$ represents the amount of incremental variance explained by each block of variables. Other tests $=$ number of other tests students had that week; ${ }^{\dagger} p<.10,{ }^{*} p<.05,{ }^{* *} p<.01,{ }^{* * *} p<.001$.

that individuals' intellectual ability is less important in determining anxiety than a subjective sense of one's ability to cope. These findings suggest that cognitive appraisals not only play an important role in emotional responses to major life events, but also predict more transient daily anxiety. Our findings also indicate that these cognitive appraisals are not merely a reflection of chronic anxiety, but rather make a unique contribution to the prediction of future anxiety in response to minor stress.

The factor score only predicted a small incremental portion of the variance in state anxiety $(1.1 \%)$ after controlling for trait anxiety and other predictors, bringing into question whether this factor makes a meaningful contribution even though it is statistically significant. Our stringent analytic approach gives priority to trait anxiety and the other predictors in accounting for the variance in state anxiety. It is also likely that our analysis underestimates the importance of the confidence factor, because these cognitive appraisals should contribute to development of chronic levels of trait anxiety. Given that this factor predicts transient state anxiety responses under these rigorous testing conditions with trait anxiety statistically controlled, it is likely that confidence appraisals are not only significant but also meaningful as predictors of responses to stress.

An important question to consider is how the cognitive appraisals represented in the confidence factor may reduce risk for anxiety. According to the framework proposed by Lazarus and Folkman (1984), these appraisals ultimately influence an individual's psychological resilience to threat through the engagement of coping strategies. For example, if one expects bad things to happen, then the only way to reduce negative emotions in response to a threat is to engage in avoidance, whereas if one has a general sense that things will turn out well, one will 
be able to approach threats and engage in active coping strategies. Taylor and colleagues (2008) showed that psychosocial resources (which include optimism, mastery, and self-esteem) did not influence individuals' detection of threat, but enhanced individuals' ability to inhibit threat responses. Thus, it is possible that individuals with confidence are able to more effectively manage threats once they are detected by promoting effective coping strategies.

Our findings suggest that the cognitive appraisals assessed in this study may be used to identify those at risk for future anxiety and that improving these cognitive appraisals through interventions may be an effective strategy for enhancing individuals' coping responses and decreasing anxiety. That is, knowing that a person is chronically anxious is an effective predictor of response to a specific threat, but it does not point to preventive psychosocial interventions for anxiety. Previous research showed that increases in control-related beliefs significantly mediated anxiety and depressive symptom prevention for at-risk undergraduates randomly assigned to an 8-week cognitive-behavioral workshop (Seligman, Schulman, \& Tryon, 2007). Our findings suggest that early intervention with at-risk individuals may be beneficial given that these appraisals have an impact on emotional responses to life's hassles, which are known to be important predictors of psychological well-being (Monroe, 1983). The empirical overlap among the six cognitive measures also suggests that interventions targeting any of these appraisals should help to buffer against anxiety, whether bolstering optimism or self-esteem through cognitive restructuring or increasing a sense of mastery or self-efficacy through exposures.

A number of limitations should be considered when interpreting the results of this study. First, our results are consistent with a causal model in which cognitive appraisals lead to the development of anxiety; however, this does not rule out the possibility of a bi-directional relationship between these constructs or third variables that may account for this relationship. More extended prospective research and experimental studies are needed to increase confidence in these findings. Second, we also cannot establish whether state anxiety reported at Time 2 was necessarily provoked by the psychology exam, although the correlation between the number of exams and state anxiety suggests that the anxiety was exam-related. Certainly this is a disadvantage of using a more naturalistic stressor; however, it is likely that this would weaken rather than enhance our chances of finding systematic variance in state anxiety. Third, because we assessed responses to a single, relatively mild stressor, it remains unclear whether these cognitive dispositions represent risk factors for clinical levels of pathology. Fourth, we have discounted the role of intellectual ability, as measured by the SATs, as a predictor of exam anxiety. However, the SAT range in a sample of Ivy League students is limited, and these results might differ in a more heterogeneous population. Finally, it is possible that additional cognitive measures not assessed in the current study should be included as part of this latent confidence construct.

Despite these limitations, the current study has several important strengths. We examined anxiety in the context of a salient and ecologically valid stressor, which helps to increase our understanding of how these cognitive dispositions function to predict individuals' anxiety in response to more common stressors in daily life. The prospective nature of these data also suggests that this latent construct can be used to identify those at risk for anxiety and may be a viable target for intervention. Future studies should test the boundaries and meaning of this latent factor by examining more disparate cognitive appraisals and determining how this factor is associated with the engagement of coping strategies. Better understanding of the mechanisms that contribute to anxiety should enhance our ability to intervene effectively. The current study represents one step towards this goal, demonstrating that cognitive appraisals are not only associated with anxiety, but also likely play a role in the development of anxiety in daily real-world settings.

\section{Acknowledgements}

The authors wish to thank Paul McDermott for statistical consultation.

\section{REFERENCES}

Ahrens , A. H., \& Haaga, D. A. F. (1993). The specificity of attributional style and expectations to positive and negative affectivity, depression, and anxiety. Cognitive Therapy and Research, 17, 83-98. doi:10.1007/BF01172742

Archer, R. P. (1979). Relationships between locus of control and anxiety. Journal of Personality Assessment, 43, 617-626. doi:10.1207/s15327752jpa4306_10

Bárez, M., Blasco, T., Fernández-Castro, J., \& Viladrich, C. (2009). Perceived control and psychological distress in women with breast cancer: A longitudinal study. Journal of Behavioral Medicine, 32, 187-196. doi:10.1007/s10865-008-9180-5

Ben-Zur, H., \& Zeidner, M. (1988). Sex differences in anxiety, curiosity, and anger: A cross-cultural study. Sex Roles, 19, 335-347. doi:10.1007/BF00289841

Bowen, R., South, M., Fischer, D., \& Looman, T. (1994). Depression, mastery and number of group sessions attended predict outcome of patients with panic and agoraphobia in a behavioural/medication program. Canadian Journal of Psychiatry, 39, 283-288.

Clark, D. A., \& Beck, A. T. (2010). Cognitive therapy of anxiety disorders: Science and practice. New York: Guilford Press.

Dew, M. A., Becker, J. T., Sanchez, J., Caldararo, R., Lopez, O. L. Wess, J. et al. (1997). Prevalence and predictors of depressive, anxiety and substance use disorders in HIV-infected and uninfected men: A longitudinal evaluation. Psychological Medicine, 27, 395-409. doi:10.1017/S0033291796004552

Geweke, J. F., \& Singleton, K. I. (1980). Interpreting the likelihood of ratio statistic in factor models when sample size is small. Journal of American Statistical Association, 75, 133-137. doi: $10.2307 / 2287400$

Gray-Little, B., Williams, V. S. L., \& Hancock, T. D. (1997). An item response theory analysis of the Rosenberg self-esteem scale. Personality and Social Psychology Bulletin, 23, 443-451. doi:10.1177/0146167297235001

Gurung, R. A. R., Dunkel-Schetter, C., Collins, N. J., Rini, C., \& Hobel, C. J. (2005). Psychosocial predictors of prenatal anxiety. Journal of Social and Clinical Psychology, 24, 497-519. doi:10.1521/jscp.2005.24.4.497

Judge, T. A., Erez, A., Bono, J. E., \& Thoresen, C. J. (2002). Are measures of self-esteem, neuroticism, locus of control, and generalized self-efficacy indicators of a common core construct? Journal of Personality and Social Psychology, 83, 693-710. doi:10.1037/0022-3514.83.3.693

Judge, T. A., Locke, E. A., \& Durham, C. C. (1997). The dispositional causes of job satisfaction: A core evaluations approach. Research in Organizational Behavior, 19, 151-188.

Lazarus, R. S., \& Folkman, S. (1984). Stress, appraisal, and coping. New York: Springer.

Lee, A., \& Hankin, B. L. (2009). Insecure attachment, dysfunctional attitudes, and low self-esteem predicting prospective symptoms of depression and anxiety during adolescence. Journal of Clinical Child 
and Adolescent Psychology, 38, 219-231. doi:10.1080/15374410802698396

Luszczynska, A., Scholz, U., \& Schwarzer, R. (2005). The general self-efficacy scale: Multicultural validation studies. The Journal of Psychology, 139, 439-457. doi:10.3200/JRLP.139.5.439-457

Monroe, S. M. (1983). Major and minor life events as predictors of psychological distress: Further issues and findings. Journal of Behavioral Medicine, 6, 189-205. doi:10.1007/BF00845380

Pearlin, L. I., Menaghan, E. G., Lieberman, M. A., \& Mullen, J. T. (1981). The stress process. Journal of Health and Social Behavior, 22, 337-356. doi:10.2307/2136676

Pearlin, L. I., \& Schooler, C. (1978). The structure of coping. Journal of Health and Social Behavior, 19, 2-21. doi:10.2307/2136319

Peterson, C., Semmel, A., von Baeyer, C., Abramson, L. Y., Metalsky, G. I., \& Seligman, M. E. P. (1982). The attributional style questionnaire. Cognitive Therapy and Research, 6, 287-299. doi:10.1007/BF01173577

Rosenberg, M. (1965). Society and the adolescent self-image. Princeton, NJ: Princeton University Press.

Rotter, J. B. (1966). Generalized expectancies for internal versus external control of reinforcement. Psychological Monographs, 80.

Sanderson, W. C., Rapee, R. M., \& Barlow, D. H. (1989). The influence of an illusion of control on panic attacks induced via inhalation of 5.5\% carbon dioxide-enriched air. Archives of General Psychiatry, 46, 157-162. doi:10.1001/archpsyc.1989.01810020059010

Scheier, M. F., \& Carver, C. S. (1992). Effects of optimism on psychological and physical well-being: Theoretical overview and empirical update. Cognitive Therapy and Research, 16, 201-228. doi:10.1007/BF01173489

Scheier, M. F., Carver, C. S., \& Bridges, M. W. (1994). Distinguishing optimism from neuroticism (and trait anxiety, self-mastery, and self-esteem): A reevaluation of the Life Orientation Test. Journal of Personality and Social Psychology, 67, 1063-1078. doi:10.1037/0022-3514.67.6.1063

Scheier, M. F., Carver, C. S., \& Bridges, M. W. (2001). Optimism, pessimism, and psychological well-being. In E. C. Chang (Ed.), Optimism and pessimism: Implications for theory, research, and practice (pp. 189-215). Washington, DC: American Psychological Assocaition. doi:10.1037/10385-009

Schwarzer, R., \& Jerusalem, M. (1995). Generalized self-efficacy scale. In J. Weinman, S. Wright, \& M. Johnston (Eds.), Measures in health psychology: A user's portfolio. Causal and control beliefs (pp. 35-37). Windsor, UK: NFER-NELSON.

Seligman, M. E., Schulman, P., \& Tryon, A. M. (2007). Group prevention of depression and anxiety symptoms. Behaviour Research and Therapy, 45, 1111-1126. doi:10.1016/j.brat.2006.09.010

Souza, T. M., Samaraweera, N., \& Frye, L. A. (2009). Anxiety and PTSD in the 2009-2010 college population. Anxiety Disorders: A Tri-Annual Report, 6, 1-6. http://www.anxsig.org/sites/default/files/newsletters/2009\%20Summ er\%20ANXSIG.pdf

Spielberger, C. D., Gorsuch, R. L., Lushene, R. E., Vagg, P. R., \& Jacobs, G. A. (1983). Manual for the state-trait anxiety inventory. Palo Alto, CA: Consulting Psychologists Press.

Taylor, S. E., Burklund, L. J., Eisenberger, N. I., Lehman, B. J., Hilmert, C. J., \& Lieberman, M. D. (2008). Neural bases of moderation of cortisol stress responses by psychosocial resources. Journal of Personality and Social Psychology, 95, 197-211. doi:10.1037/0022-3514.95.1.197

van Jaarsveld, C. H., Ranchor, A. V., Sanderman, R., Ormel, J., \& Kempen, G. I. (2005). The role of premorbid psychological attributes in short- and long-term adjustment after cardiac disease. A prospective study in the elderly in The Netherlands. Social Science \& Medicine, 60, 1035-1045. doi:10.1016/j.socscimed.2004.06.037

Windsor, T. D., Anstey, K. J., Butterworth, P., Luszcz, M. A., \& Andrews, G. R. (2007). The role of perceived control in explaining depressive symptoms associated with driving cessation in a longitudinal study. The Gerontologist, 47, 215-223. doi:10.1093/geront/47.2.215 